\title{
Low-Blood Lymphocyte Number and Lymphocyte Decline as Key Factors in COPD Outcomes: A Longitudinal Cohort Study
}

\author{
Umberto Semenzato $^{a}$ Davide Biondini $^{a} \quad$ Erica Bazzan $^{a}$ Mariaenrica Tinéa \\ Elisabetta Balestro $^{a}$ Barbara Buldini ${ }^{b}$ Santiago J. Carizzo ${ }^{c, d}$ Pablo Cubero ${ }^{e}$ \\ Marta Marin-Oto ${ }^{\mathrm{e}} \mathrm{f} \quad$ Alvise Casara $^{\mathrm{a}}$ Simonetta Baraldo ${ }^{\mathrm{a}}$ Graziella Turato ${ }^{a}$ \\ Dario Gregori ${ }^{a}$ Jose M. Marin c, d, e Manuel G. Cosio ${ }^{a, g}$ Marina Saetta $^{a}$ \\ aDepartment of Cardiac, Thoracic, Vascular Sciences and Public Health, University of Padova, Padova, Italy; \\ bDepartment of Women's and Children's Health, University of Padova, Padova, Italy; 'Servicio de Neumología, \\ Hospital Universitario Miguel Servet, IISAragon, Zaragoza, Spain; ${ }^{\mathrm{d} C I B E R}$ Enfermedades Respiratorias, Madrid, \\ Spain; eUnidad de Investigación Traslacional, IISAragon, Zaragoza, Spain; fDepartamento de Neumología, Clinico \\ Universidad de Navarra, Pamplona, Spain; ${ }^{9}$ Respiratory Division, Meakins-Christie Laboratories, McGill University, \\ Montreal, QC, Canada
}

\section{Keywords}

COPD outcomes · Lymphocyte decline $\cdot$ COPD survival

\begin{abstract}
Background: Smokers with and without chronic obstructive pulmonary disease (COPD) are at risk of severe outcomes like exacerbations, cancer, respiratory failure, and decreased survival. The mechanisms for these outcomes are unclear; however, there is evidence that blood lymphocytes (BL) number might play a role. Objective: The objective of this study is to investigate the relationship between $\mathrm{BL}$ and their possible decline over time with long-term outcomes in smokers with and without COPD. Methods: In 511 smokers, 302 with COPD (COPD) and 209 without COPD (noCOPD), followed long term, we investigated whether $\mathrm{BL}$ number and $\mathrm{BL}$ decline over time might be associated with long-term outcomes. Smokers were divided according to $\mathrm{BL}$ number in high-BL $(\geq 1,800$ cells $/ \mu \mathrm{L})$ and low-BL $(<1,800$ cells $/ \mu \mathrm{L})$. Clinical features, cancer incidence, and mortality were recorded during
\end{abstract}

follow-up. BL count in multiple samples and $\mathrm{BL}$ decline over time were calculated and related to outcomes. Results: BL count was lower in COPD $(1,880$ cells $/ \mu \mathrm{L})$ than noCOPD $(2,300$ cells $/ \mu \mathrm{L} ; p<0.001) .43 \%$ of COPD and $23 \%$ of noCOPD had low-BL count $(p<0.001)$. BL decline over time was higher in COPD than noCOPD ( $p=0.040)$. $22.5 \%$ of the whole cohort developed cancer which incidence was higher in low-BL subjects and in $\mathrm{BL}$ decliners than high-BL (31 vs. $18 \% ; p=$ 0.001 ) and no decliners (32 vs. $19 \% ; p=0.002$ ). $26 \%$ in the cohort died during follow-up. Furthermore, low-BL count, BL decline, and age were independent risk factors for mortality by Cox regression analysis. Conclusion: BL count and BL decline are related to worse outcomes in smokers with and without COPD, which suggests that $\mathrm{BL}$ count and decline might play a mechanistic role in outcomes deterioration. In-

Drs. Semenzato and Biondini contributed equally to this article as first authors.

Profs. Saetta and Cosio contributed equally to this article as senior authors.
(C) 2021 The Author(s)

Published by S. Karger AG, Basel

This is an Open Access article licensed under the Creative Commons Attribution-NonCommercial-4.0 International License (CC BY-NC) (http://www.karger.com/Services/OpenAccessLicense), applicable to the online version of the article only. Usage and distribution for commercial purposes requires written permission.
Correspondence to:

Marina Saetta, marina.saetta@ unipd.it 
sights into mechanisms inducing the fall in $\mathrm{BL}$ count could improve the understanding of COPD pathogenesis and point toward new therapeutic measures.

(C) 2021 The Author(s)

Published by S. Karger AG, Basel

\section{Introduction}

Cigarette smoke is the major risk factor for the development of chronic obstructive pulmonary disease (COPD), the third leading cause of death worldwide [1]. The disease is characterized by an abnormal innate and adaptive immune inflammation comprising neutrophils, monocytes, and T and B lymphocytes [2-4], which is responsible for the airway and parenchymal abnormalities leading to the progressive airflow limitation and respiratory failure, characteristic of the disease [1].

Because lymphocytes traffic between inflammatory sites, lymph nodes, and systemic circulation [5], the idea that cigarette smoke causes not only lung but also systemic inflammation with consequent comorbid effects has been pursued $[6,7]$. In favor of this possibility was the description in 1971 that cigarette smoking was associated with an increase in blood leukocytes which was related to the amount smoked [8].

The eventual awareness of the role of lymphocytes in COPD pathogenesis [9-11] prompted numerous studies assessing the possible associations of peripheral blood lymphocytes (BL) with smoking and COPD. These investigations established that the absolute lymphocyte number was increased in smokers, with a tendency toward increased $\mathrm{CD} 4^{+}$but not $\mathrm{CD} 8^{+} \mathrm{T}$ cells and a slightly increased $\mathrm{CD} 4^{+} / \mathrm{CD}^{+}$ratio $[12,13]$. Conversely, in $\mathrm{COPD}, \mathrm{CD} 4^{+} \mathrm{T}$ cells were lower than in normal subjects, while $\mathrm{CD} 8^{+} \mathrm{T}$ cells were similar with a decreased $\mathrm{CD} 4^{+} / \mathrm{CD}^{+}$ratio [14-16]. Yet the potential effects of the lymphocyte changes in COPD clinical outcomes and survival were never assessed. It is now evident that a decrease in the absolute BL count is associated with subsequent mortality in the "healthy elderly" and also with poor prognosis and increased mortality in other conditions like connective tissue diseases, sarcoidosis, bacterial infections, congestive heart failure, and cancer $[17,18]$.

In the last few years, the ratio of $\mathrm{BL}$ in relation to neutrophils (the neutrophil-to-lymphocyte ratio (NLR)) has been used as a possible biomarker for inflammation in COPD [19]. An association between NLR and disease severity, exacerbations, and higher hospitalization rate has been reported [19].

Blood Lymphocytes in COPD
More recently, in COPD, BL have been analyzed alone since increases in NLR are most often due to low-lymphocyte count, and lymphocytes are not as much influenced by acute events as the neutrophils. A recent post hoc study showed that a single determination of BL, monocytes, and eosinophils was predictive of 3-year change in lung function, with high lymphocytes being predictive of a better functional outcome [20]; however, other long-term outcomes like severe exacerbations, cancer, and mortality were not evaluated. In another recent study, it has been reported that a low lymphocyte proportion in peripheral blood was associated with higher mortality in elderly people with severe COPD [21].

Based on these findings, we wanted to investigate the relationship between BL and their possible decline over time with long-term outcomes, including cancer development and mortality, in smokers without and with mild-to-severe COPD [22]. For this purpose, we used an ongoing cohort of smokers with COPD (COPD) and without COPD (noCOPD), free of significant comorbid conditions at recruitment, followed longitudinally for 5 years.

\section{Methods}

Study Population

Participants were recruited among smokers who first attended the Pulmonary Clinic at the Hospital Universitario Miguel Servet (Zaragoza, Spain) requesting to be included in a smoking cessation program or referred by other doctors to assess their respiratory health between October 2010 and April 2014. The inclusion and exclusion criteria are detailed in online suppl. Fig. 1; for all online suppl. material, see www.karger.com/doi/10.1159/000515180.

At baseline, all subjects were clinically stable, free of major comorbidities, not having had any exacerbations or oral corticosteroid and antibiotic therapy for at least 8 weeks. Subjects with asthma or history of asthma, bronchiectasis, autoimmune diseases, hematological diseases, other respiratory diseases or coexisting malignancy at recruitment were excluded. This study was approved by human-research review board (IRB.12/2010), and all patients provided informed written consent before any procedure was done.

\section{Measurements and Follow-Up}

At baseline, subjects underwent a comprehensive functional and clinical examination (online suppl. data). All subjects had a prior lung function test and blood cell count obtained in stable conditions before study entry. COPD was defined by $\mathrm{FEV}_{1} / \mathrm{FVC}$ $<0.70$ post-bronchodilator, and severity was defined by GOLD spirometry criteria [1]. Complete blood cell count was measured at baseline and at every follow-up visit, which was done in stable conditions approximately every year. The change in BL number over time was calculated in all subjects comparing the first and the last sample as described by Lin et al. [23]. Subjects in whom BL 
Table 1. Clinical and functional characteristics of all smokers, noCOPD and COPD

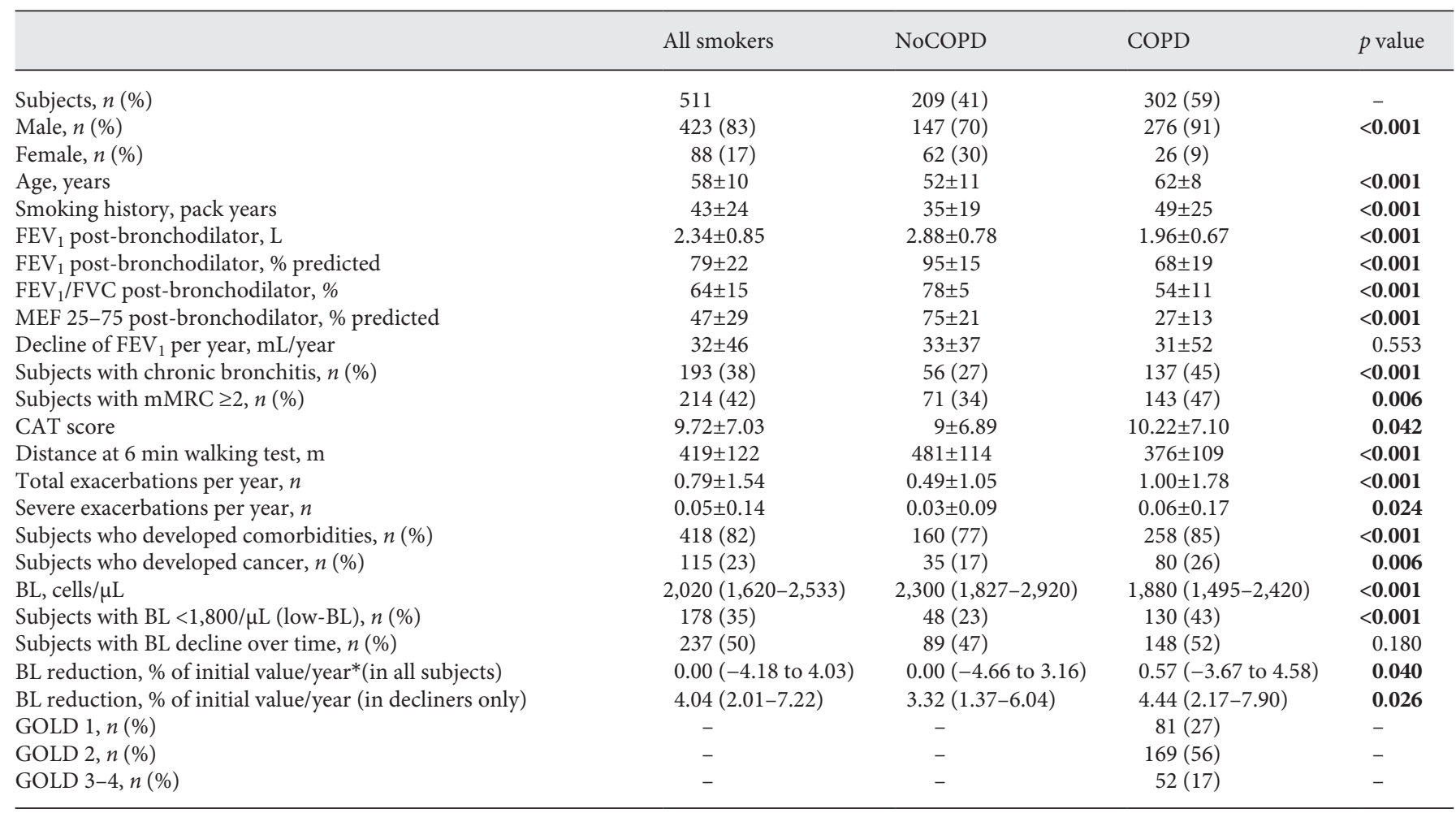

Data are presented as $n(\%)$, mean $\pm \mathrm{SD}$, or median (interquartile range), $p$ value refers to Mann-Whitney test or $\chi^{2}$ test, for comparisons between noCOPD and COPD. Significant $p$ values in bold. COPD, chronic obstructive pulmonary disease; noCOPD, without COPD; COPD, with COPD; BL, blood lymphocytes. * Negative values mean gain.

number had a decrease of at least $1 \%$ per year compared to the initial value in at least 3 years of follow-up, were classified as decliners. In a subgroup of 18 subjects, 8 COPD and 10 noCOPD, the percentages of $\mathrm{T}$-CD3 ${ }^{+}$, T-helper $\left(\mathrm{CD}^{+} \mathrm{CD}^{+}\right)$, T-cytotoxic $\left(\mathrm{CD}^{+}{ }^{\mathrm{CD}} 8^{+}\right), \mathrm{B}\left(\mathrm{CD} 3^{-} \mathrm{CD} 19^{+}\right)$, and $\mathrm{NK}$ cells $\left(\mathrm{CD} 3^{-} \mathrm{CD} 16^{+} \mathrm{CD} 56^{+}\right)$ in peripheral blood were determined by flow cytometry (online suppl. data).

The annual frequency and type of exacerbations, as defined by recent guidelines [1], were collected at every follow-up visit. The incidence of comorbidities, including cancer, was prospectively recorded at every follow-up visit; all-cause mortality and cause of death were recorded up to October 2018.

\section{Statistical Analysis}

Comparisons among groups were evaluated with KruskalWallis and Mann-Whitney U tests. Distributions of categorical variables were compared with the $\chi^{2}$-test. Logistic regression analysis was used to adjust for potential confounding factors. In order to confirm if BL sequentially declined from baseline in every follow-up visit, the Friedman's test for repeated measures was used. Analyses of overall survival were performed using Kaplan-Meier survival curves. Multivariate Cox proportional hazard regression model was used to evaluate independent prognostic factors, after adjusting for all non-time dependent and time dependent covariates (online suppl. data).

\section{Results}

\section{Demographic and Clinical Characteristics}

Of the 1,323 eligible smokers seen in outpatient clinic, 511 [302 COPD and 209 noCOPD] met the inclusion criteria for the study (in the online suppl. Fig. 1). COPD were older, smoked more, and had lower $\mathrm{FEV}_{1}$ than noCOPD. COPD had higher prevalence of chronic bronchitis, had more dyspnea and had more exacerbations than noCOPD (Table 1). The mean follow-up time for the whole cohort was $5.25 \pm 1.79$ years.

\section{Blood Lymphocyte Count}

The absolute BL number in each of the available samples was lower in COPD than in noCOPD. Figure 1a shows that the BL count in every blood sample $(1,2,3,4$, and 5) were significantly lower in each COPD sample than the corresponding sample in noCOPD $(p<0.001)$. The mean of all samples in each patient was used for statistical analysis.

Figure $1 \mathrm{~b}$ and online suppl. Figure 2 show that the median BL count in COPD $(1,880$ cells/ $\mu \mathrm{L})$, even if within 


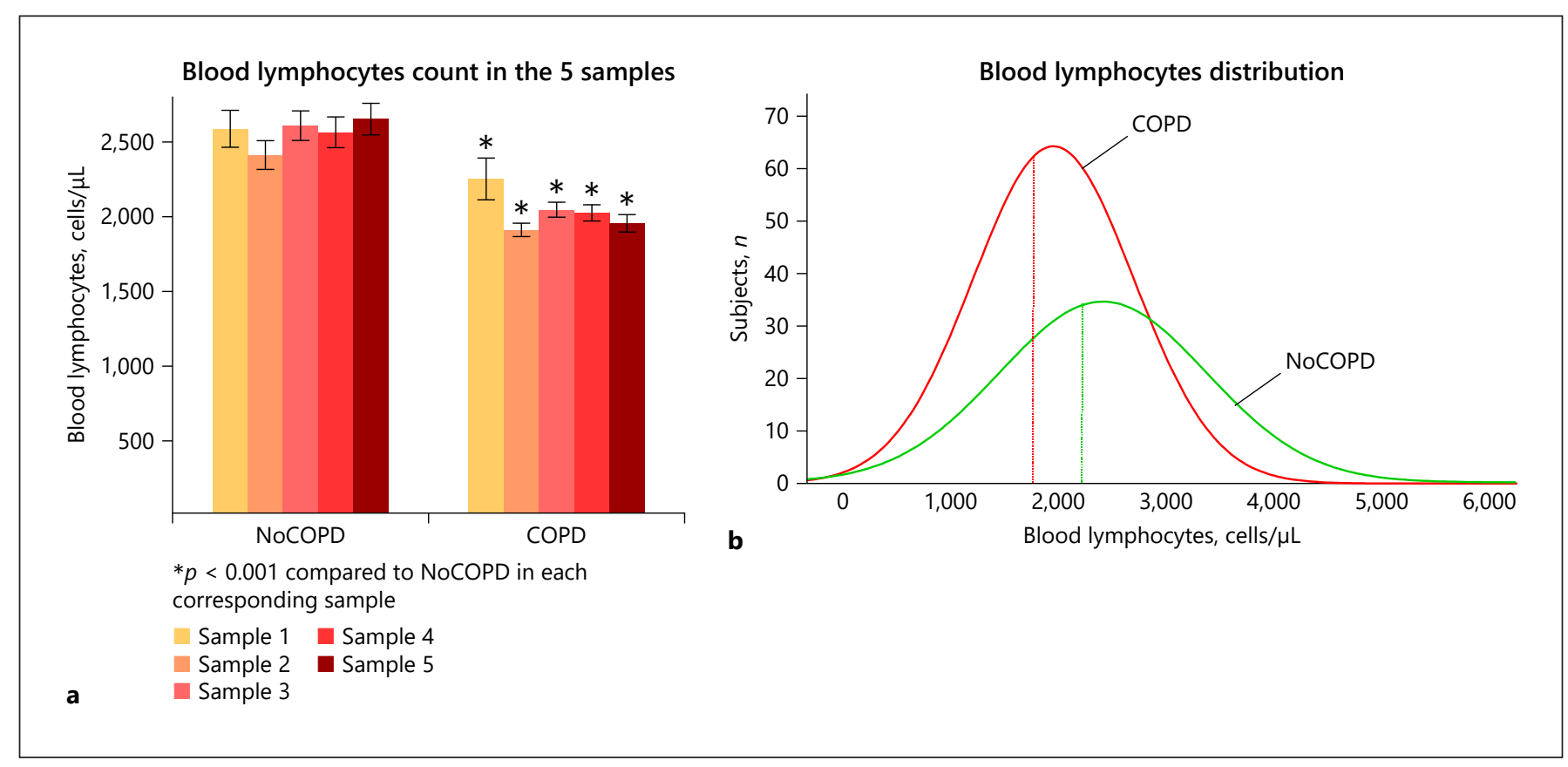

Fig. 1. BL count and distribution. (a) Mean BL count at each of the 5 blood samples in COPD and noCOPD (noCOPD number of patients: Sample $1 n=209$, Sample $2 n=209$, Sample $3 n=188$, Sample $4 n=173$, and Sample $5 n=153$; COPD: Sample $1 n=302$, Sample $2 n=302$, Sample $3 n=285$, Sample $4 n=262$, and Sample $5 n=235)$. *indicates a significant lower BL number in each COPD blood sample than the corresponding noCOPD sample $\left({ }^{*} p<0.001\right.$ for every sample comparison). (b) Distribution of BL (mean of all samples) in COPD (red line) and noCOPD (green line); vertical lines show median in COPD and noCOPD (1,880 vs. 2,300 cells/ $\mu \mathrm{L} ; p<0.001)$. COPD, chronic obstructive pulmonary disease; noCOPD, without COPD; COPD, with COPD; BL, blood lymphocyte.

\section{Change in Blood Lymphocyte Count over Time}

For the calculation of BL decline in our population, we count in noCOPD $(2,300$ cells/ $\mu \mathrm{L} ; p<0.001)$. In order to have an easier to handle number as a cutoff, we rounded the COPD median BL count of 1,880 cells/ $\mu \mathrm{L}$ to 1,800 cells/ $\mu \mathrm{L}$, a value used before in another publication [24]. The value of 1,800 cells $/ \mu \mathrm{L}$ was used as cutoff to define low-BL $(<1,800$ cells $/ \mu \mathrm{L})$ and high-BL $(\geq 1,800$ cells $/ \mu \mathrm{L})$. $43 \%$ of COPD and $23 \%$ of noCOPD had low-BL count $(p<0.001$; Table 1$)$. There was no difference in lymphocyte count in peripheral blood between active smokers and former smokers neither in the whole population nor in the noCOPD and COPD groups $(p=0.195)$ (online suppl. data). Among COPD subjects, there was no difference in BL count between inhaled corticosteroids-treated and nontreated patients $(p=0.288)$. BL flow cytometry analysis showed that COPD with low-BL had lower percentages of $\mathrm{TCD}^{+}$and $\mathrm{B}$ lymphocytes, increased percentages of $\mathrm{TCD}^{+}$and NK cells, and lower $\mathrm{CD} 4^{+} / \mathrm{CD} 8^{+}$ ratio than COPD with high-BL and noCOPD (online suppl. Fig. 3). 
Table 2. Clinical and functional characteristics of noCOPD and COPD with low-BL $(<1,800 / \mu \mathrm{L})$ and high-BL $(\geq 1,800 / \mu \mathrm{L})$

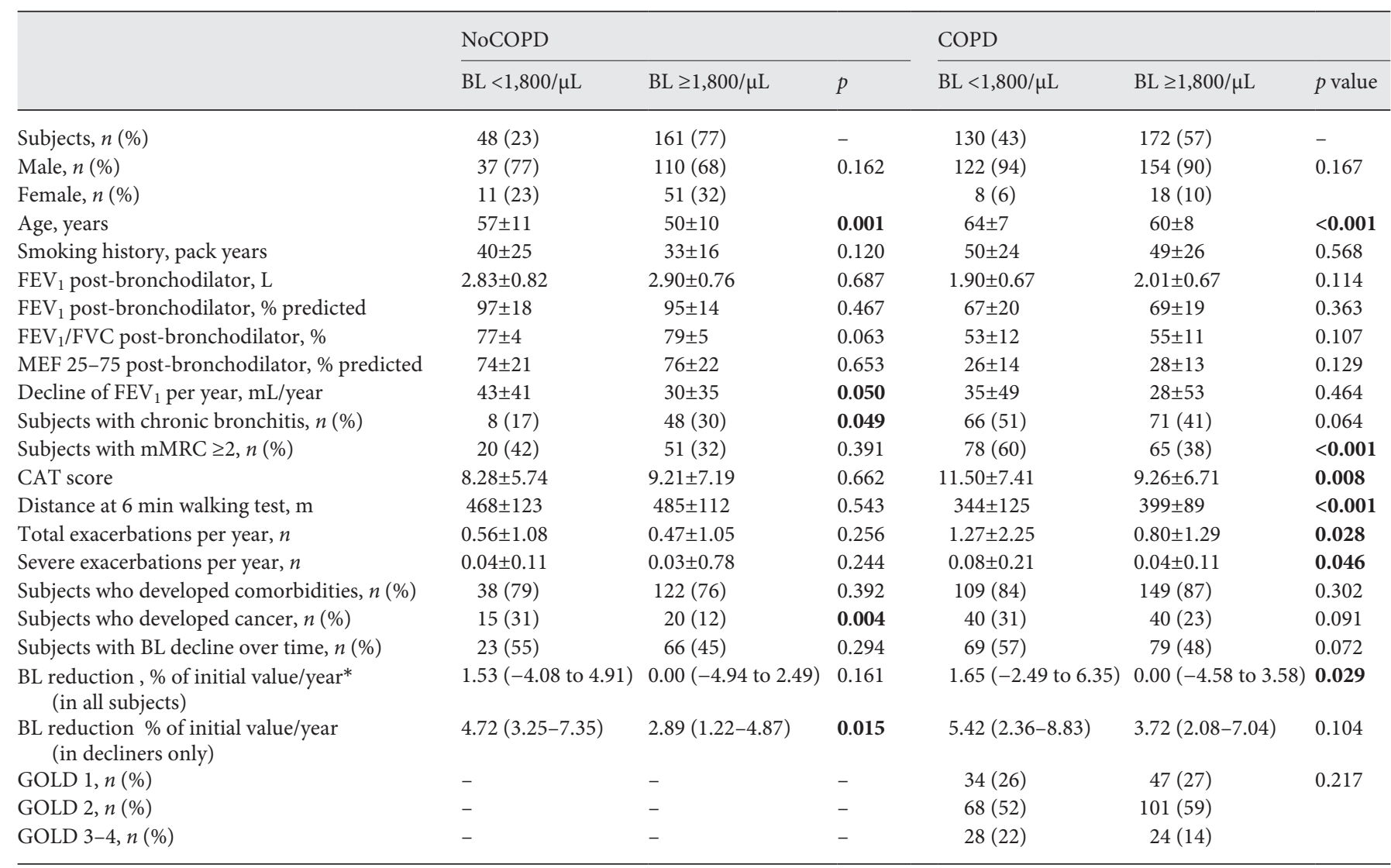

Data are presented as $n(\%)$ or mean $\pm \mathrm{SD}$, $p$ value refers to Mann-Whitney test or $\chi^{2}$ test. Significant $p$ values in bold. COPD, chronic obstructive pulmonary disease; noCOPD, without COPD; COPD, with COPD; BL, blood lymphocytes. * Negative values mean gain.

In both COPD and noCOPD, BL count decreased over time in about $50 \%$ of subjects (decliners) and increased or remained stable in the other half (no decliners). The $\mathrm{BL}$ decline was higher in all COPD cases than in all noCOPD ( $p=0.040)$, and this difference was magnified when only decliners were considered $(p=0.026$; Table 1$)$.

\section{Blood Lymphocyte Count and Clinical Characteristics}

Table 2 shows the characteristics of COPD and noCOPD with low or high BL. NoCOPD with low-BL were older and had higher $\mathrm{FEV}_{1}$ decline than noCOPD with high-BL ( $p=0.001$ and $p=0.05$, respectively). COPD with low-BL, in spite of similar lung function, were more dyspneic, had higher CAT score and performed worse in the 6 -min walking test than COPD with high-BL $(p<0.001$, $p=0.008, p<0.001$, respectively; online suppl. Fig. 4). In COPD, BODE index was significantly higher in subjects with low-BL than those with high-BL (2.8 \pm 2.4 vs. $1.7 \pm$
$1.6 ; p<0.001)[25]$. BL numbers were similar in all GOLD stages.

In COPD, 53\% $(n=161)$ exacerbated at least once during the follow-up and those with low-BL had more exacerbations than those with high-BL (1.27 vs. 0.80 exacerbations/year; $p=0.028$ ) (Table 2 ; online suppl. Fig. 5). COPD developed more comorbidities other than cancer (85\%) than noCOPD (77\%; Table 1$)$ with no relation to BL count (Table 2).

When these analyses were performed using only the baseline BL value instead of the mean of all 5 samples, the main results were confirmed. In particular, subjects with COPD and low-BL at baseline were more dyspneic, had higher CAT score, performed worse in the 6-min walking test, and had a higher BODE index than COPD with high$\mathrm{BL}(p=0.013, p=0.022, p=0.028, p=0.023$, respectively). COPD with low-BL had also more exacerbations than those with high-BL $(p=0.049)$. 


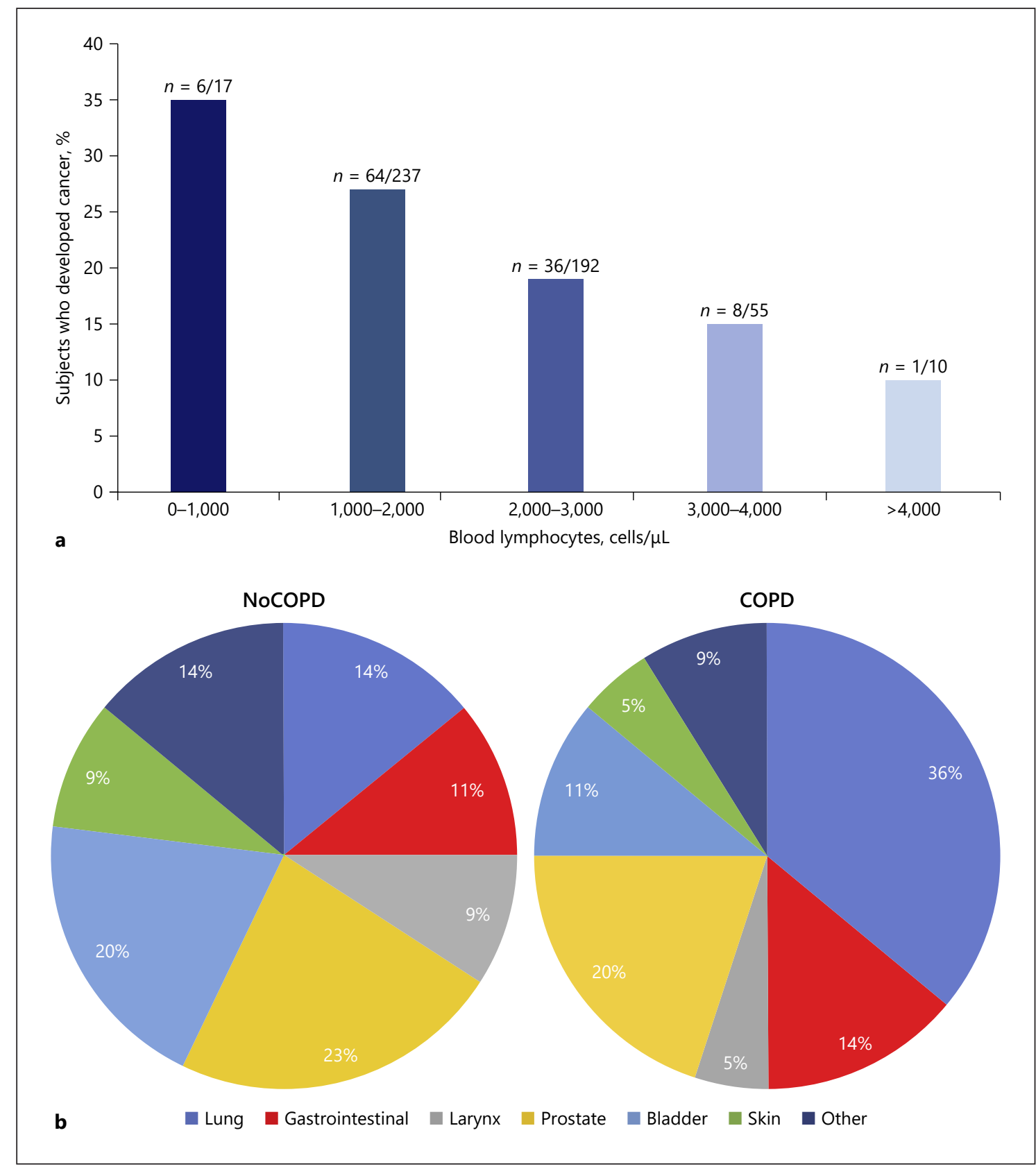

Fig. 2. Incidence of cancer in the study cohort. (a) Incidence of cancer according to BL count. Percentage of subjects who developed all types of cancer according to intervals of BL number (from 0 to 1,000 cells $/ \mu \mathrm{L}$ to $>4,000$ cell $/ \mu \mathrm{L} ; \chi^{2}$-test, $p=0.004$ among all intervals). (b) Percent of the different types of cancer developed by the 35 noCOPD and the $80 \mathrm{COPD}$. The most frequent cancers

\section{Blood Lymphocyte Count and Cancer}

In our cohort, 115 of 511 subjects (22.5\%) developed cancer during follow-up. The incidence was higher in COPD than in noCOPD (26 vs. $17 \%, p=0.006$; Table 1 ). were prostate $(n=8)$, bladder $(n=7)$, and lung $(n=5)$ in noCOPD and lung $(n=29)$, prostate $(n=16)$, and gastrointestinal $(n=11)$ in COPD. COPD, chronic obstructive pulmonary disease; noCOPD, without COPD; COPD, with COPD; BL, blood lymphocytes. 
There was an important inverse relation between $\mathrm{BL}$ count and cancer incidence, so as BL number decreased, cancer incidence increased ( $p=0.004$; Fig. $2 \mathrm{a}$ ).

In the whole cohort, subjects with low-BL had a higher cancer incidence than those with high-BL (31 vs. $18 \%$; $p=0.001$ ). A higher cancer incidence was also observed in BL decliners compared to no decliners (32 vs. 19\%; $p=$ 0.002). In noCOPD, subjects with low-BL had a higher incidence of any type of cancer ( 31 vs. $12 \% ; p=0.004$ ) and lung cancer ( 10 vs. $0 \% ; p=0.001$ ) than subjects with highBL. Subjects with BL decline had a higher incidence of any type of cancer than no decliners (22 vs. $14 \%$; $p=0.040$ ). In COPD, the incidence of any type of cancer (32 vs. 19\%; $p=0.002$ ) and of lung cancer (14 vs. $5 \% ; p=0.011$ ) was higher in BL decliners than in no decliners. The proportion of the 88 females in the cohort developing lung cancer $(6.8 \%)$ was similar to that of males $(6.6 \%)$, while a higher percentage of males (17.9\%) than females (5.6\%) developed other cancers.

\section{Blood Lymphocyte Count and Survival}

134 subjects (26\%) died during follow-up (114 COPD and 20 noCOPD): $41 \%$ from respiratory causes, $19 \%$ from lung cancer, $12 \%$ from cardiovascular diseases and $28 \%$ from the other causes (online suppl. Fig. 6). KaplanMeier analysis showed that COPD and noCOPD with low-BL had a worse survival than those with high-BL (hazard ratio [HR] 3.26, 95\% confidence interval [CI] 2.21-4.83 $p<0.001$ in COPD; HR 3.59, 1.43-9.06, $p=$ 0.004 in noCOPD; Fig. 3). Similarly, BL decliners had worse survival than no decliners (HR 2.54, 95\% CI 1.67$3.85, p<0.001$ in COPD; HR 4.52, 95\% CI 1.48-13.74, $p=0.004$ in noCOPD; Fig. 3). Importantly, subjects with low-BL who also decline have worse survival than subjects with low-BL who do not decline $(p<0.01$ for both COPD and noCOPD).

Cox regression analysis showed that among the variables related to survival, low-BL count (HR 2.477, 95\% CI $1.625-3.777, p<0.001$ ) and BL decline (HR 6.383, 95\% CI $1.888-21.573, p=0.003)$ were independent risk factors for all-cause mortality in COPD; in noCOPD, low-BL count (HR 3.524, 95\% CI 1.239-10.019, $p=0.018$ ) and BL decline (HR 4.937, 95\% CI 1.495-16.302, $p=0.009$ ) were also risk factors for all-cause mortality (Table 3). When survival analysis was performed considering only the $\mathrm{BL}$ value at baseline, all results were confirmed, showing that COPD and noCOPD subjects with low-BL had a worse survival than those with high-BL $(p=0.011$ and $p=0.024$, respectively).

\section{Lymphocyte Count and Decline}

The 4 quadrants (A-B-C-D) in Figure 4 illustrate the determinant role of blood Lymphocyte Count and its Decline over time (LymphoCoDe) in disease outcomes. In COPD, going from stage A (high-BL no decay) to stage $\mathrm{D}$ (low-BL with decay) symptoms, incidences of cancer $\left(\chi^{2}-\right.$ test $p=0.003$, and 0.008 , respectively) and survival (Kaplan-Meier $p<0.001$ ) progressively worsen. Progression from $\mathrm{A}$ to $\mathrm{D}$ is independent from the functional GOLD stage. In noCOPD, the incidences of cancer $(p=0.001)$ and survival $(p<0.001)$ also progressively worsen from A to $\mathrm{D}$.

\section{Discussion}

In an attempt to clarify the possible role of blood lymphocytes in COPD outcomes, we quantified total BL number and rate of decline in smokers with COPD and without COPD followed long-term and related BL count to outcomes in our population. Our results showed that COPD, compared to noCOPD, have significantly lower numbers of $\mathrm{BL}$, which are associated with significantly worse outcomes including functional decay, number of exacerbations, cancer incidence, and reduced survival. We also found that the decline in BL count over time had an additive effect on the worsening outcomes associated with low-BL number. These findings point toward a possible mechanistic role of lymphocyte changes in the progression of COPD.

BL number in normal populations (range 1,100-4,800, median 2,100 cells $/ \mu \mathrm{L}$ ) exhibits a great degree of interindividual variation, and it is unclear whether these differences are due to individual's characteristics that remain stable with aging or to possible environmental or other factors $[23,26]$. The distribution of absolute BL number in our population is in keeping with the known normal BL variability; however, COPD had a systematic reduction in BL number and, as a result, $43 \%$ of COPD had low-BL $(<1,800$ cells $/ \mu \mathrm{L})$ compared to only $23 \%$ noCOPD.

One of the hallmark alterations in the immune system with age is the change in number of the different types of lymphocytes in the circulation $\left(\mathrm{CD}^{+}, \mathrm{CD} 8^{+}, \mathrm{B}\right.$ and NKcells), with a rate of change that could be either positive or negative $\left(\mathrm{CD}^{+}{ }^{+}\right.$ranging from -120 to +170 cells $/ \mu \mathrm{L} /$ year), which is quite heterogeneous across individuals but relatively stable in the same individual [23]. BL decline and its effects on disease outcomes have never been investigated. 


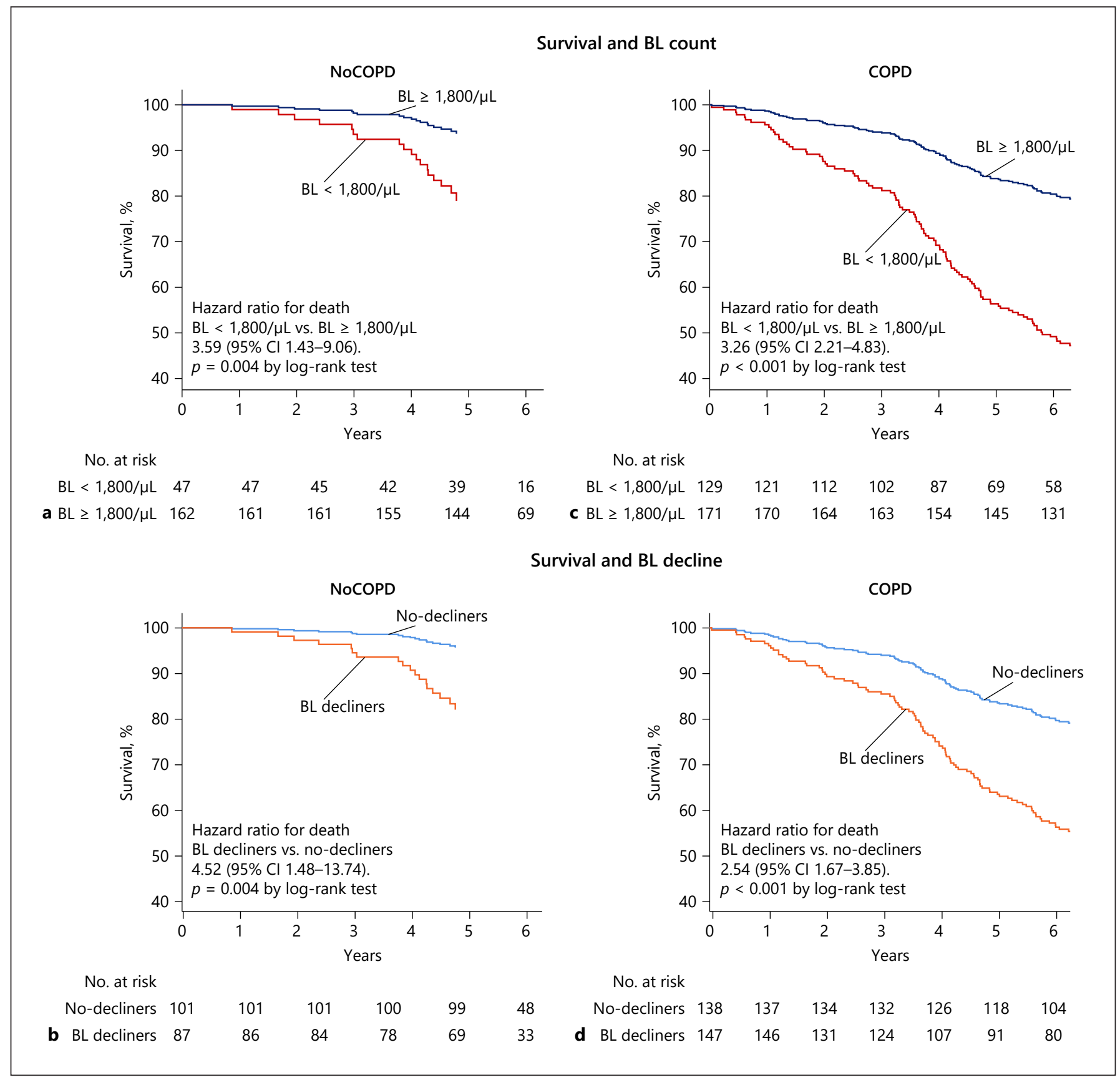

Fig. 3. Survival plots in relation to BL count and lymphocyte decline. Kaplan-Meier plots showing survival (all-cause mortality) in relation to BL count and decline during follow-up. (a) NoCOPD with low-BL had worse survival than those with high-BL ( $p=$ 0.004); (b) NoCOPD with BL decline had lower survival than no decliners $(p=0.004)$; (c) COPD with low-BL had worse survival than those with high-BL $(p<0.001)$; (d) COPD with BL decline had lower survival than no decliners $(p<0.001)$. COPD, chronic obstructive pulmonary disease; noCOPD, without COPD; COPD, with COPD; BL, blood lymphocytes.
Interestingly in our study COPD, whose BL was initially lower, showed a significantly faster decline in $\mathrm{BL}$ than noCOPD, an effect independent of age and amount smoked. These findings suggest that either subjects with an original fast rate of decay, and consequent low-BL, might be more prone to develop COPD or that, conversely, the development of COPD could be a factor promoting faster decay in BL number. 
Table 3. Cox regression analysis for survival of noCOPD and COPD

\begin{tabular}{|c|c|c|c|c|}
\hline \multirow[t]{2}{*}{ Risk factor } & \multirow{2}{*}{$\begin{array}{l}\text { Univariate analysis } \\
p \text { value }\end{array}$} & \multicolumn{3}{|c|}{ Cox regression } \\
\hline & & HR & $95 \% \mathrm{CI}$ & $p$ value \\
\hline \multicolumn{5}{|l|}{ NoCOPD } \\
\hline Low-BL, $<1,800$ cells $/ \mu \mathrm{L}$ & $<0.001$ & 3.524 & $1.239-10.019$ & 0.018 \\
\hline BL decliners & $<0.001$ & 4.937 & $1.495-16.302$ & 0.009 \\
\hline $\mathrm{FEV}_{1}$ post-BD, $\%$ pred. & 0.071 & - & - & - \\
\hline Total exacerbations per year, $n$ & 0.48 & - & - & - \\
\hline Comorbidities, $n$ & 0.047 & - & - & - \\
\hline $\mathrm{mMRC} \geq 2$ and/or CAT $\geq 10$ & 0.25 & - & - & - \\
\hline \multicolumn{5}{|l|}{$C O P D$} \\
\hline Low-BL, $<1,800$ cells $/ \mu \mathrm{L}$ & $<0.001$ & 2.477 & $1.625-3.777$ & $<0.001$ \\
\hline BL decliners & $<0.001$ & 6.383 & $1.888-21.573$ & 0.003 \\
\hline $\mathrm{FEV}_{1}$ post-BD, $\%$ pred. & $<0.001$ & 0.989 & $0.979-1.000$ & 0.061 \\
\hline Total exacerbations per year, $n$ & 0.010 & - & - & - \\
\hline Comorbidities, $n$ & 0.776 & - & - & - \\
\hline $\mathrm{mMRC} \geq 2$ and/or $\mathrm{CAT} \geq 10$ & 0.026 & - & - & - \\
\hline
\end{tabular}

Whichever the cause, we found that worse clinical and functional outcomes in smokers are influenced by low-BL number and $\mathrm{BL}$ decline. $\mathrm{BL}$ number was related to $\mathrm{FEV}_{1}$ as shown before [20]. Furthermore, COPD with low-BL despite similar age and lung function, walked significantly lower distance in $6 \mathrm{~min}$ (6MWT) and were significantly more dyspneic at rest and during exercise (mMRC scale) than those with high-BL (online suppl. Fig. 4). These findings highlight the multiple determinants possibly contributing to the complex mechanisms of dyspnea.

In COPD, BL number also influenced the frequency of moderate and severe exacerbations, which was significantly higher in COPD with low-BL than in those with high-BL (online suppl. Fig. 5). These findings suggest that the presence of low-BL number could be a possible tool to alert physicians for the risk of exacerbations in COPD.

At the study entry, all subjects were free of comorbidities including cancer, yet one-fifth of them developed a malignancy during follow-up. Remarkably the incidence of all types of cancers, including lung, significantly and steadily decreased as the absolute number of BL increased (Fig. 2a). Compounding the effects of the low-BL number, subjects who developed cancer had a higher BL decline than those without cancer. This finding was especially relevant in subjects with COPD in whom the initial low-BL number was further decreased by a faster decline, worsening the initial low-BL value.
The association of low-BL number with cancer is well established in oncology and emphasizes the importance of preserving a pool of functioning lymphocytes in the circulation $[27,28]$. The range of $\mathrm{BL}$ count seen in most of our cases is within the considered normal range, however, a substantial number of subjects, none of whom had cancer at the study entry, had BL count well into the so-called lymphopenia grade $1(800-1,000$ cells/ $\mu \mathrm{L})$ and even grade $2(500-800$ cells $/ \mu \mathrm{L})$, as defined in the cancer literature [27]. The low-BL count along with the fast BL decline very likely have an important local but also systemic effect in cancer development in smokers and could be useful to predict its development in the lung and beyond [29]. Of interest, it has been reported that in patients with new pulmonary nodules noted on chest films, low percentile of lymphocytes averaged over a three-week period distinguished bronchogenic carcinoma patients from patients with benign lesions with 95 percent overall accuracy [30].

Of the 511 subjects in our cohort, 134 (26\%) (114 COPD, 20 noCOPD) died during the follow-up. The assessment of the effects of BL number and BL decline on all-cause mortality showed that subjects with low-BL and BL decline had a significantly higher mortality than subjects with high BL and no decliners. A recent large population study reporting an important association of mortality with BL count, confirms that mortality starts increasing when $\mathrm{BL}$ values are below 1,800 cells/ $\mu \mathrm{L}$ [26]. 


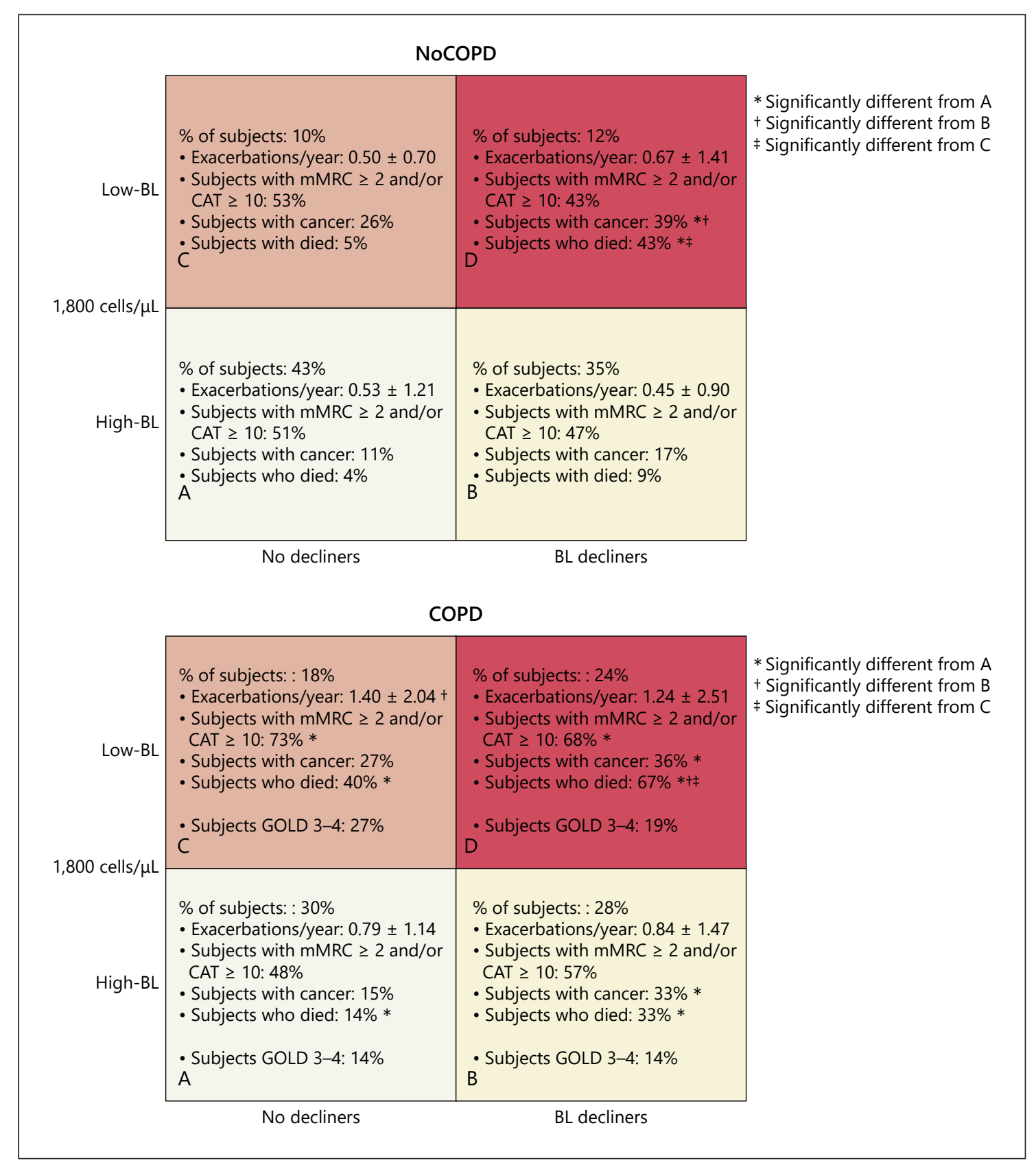

Fig. 4. Clinical outcome progression in relation to LymphoCoDe. Figure illustrates the progression of different outcomes in both noCOPD and COPD according to BL count (high-BL in A, B, low$\mathrm{BL}$ in $\mathrm{C}, \mathrm{D}$ ) and $\mathrm{BL}$ decline (no decliners in $\mathrm{A}, \mathrm{C}, \mathrm{BL}$ decliners in $B, D)$. NoCOPD: the percentage of subjects developing cancer increases significantly from $\mathrm{A}$ and $\mathrm{B}$ to $\mathrm{D}\left(\chi^{2}\right.$-test $p=0.001$ and 0.026 , respectively). Survival significantly decreases from A, B and C to $\mathrm{D}$ (Kaplan-Meier analysis, $p<0.001, p<0.001$, and $p=0.009$, respectively). COPD: exacerbations/year increase from B to $C\left(\chi^{2}-\right.$ test $p=0.041$ ). The percentage of subjects with $m M R C \geq 2$ and/or $\mathrm{CAT} \geq 10$ increases from $\mathrm{A}$ to $\mathrm{C}$ and $\mathrm{D}\left(\chi^{2}\right.$-test $p=0.004$ and 0.012 , respectively). The percentage of subjects developing cancer increases significantly from $\mathrm{A}$ to $\mathrm{B}$ and $\mathrm{D}\left(\chi^{2}\right.$-test $p=0.006$ and 0.002 , respectively). Survival significantly decreases from A, B and C to $\mathrm{D}$ (Kaplan-Meier analysis, $p<0.001, p=0.002$ and $p=0.006$, respectively), and also in the intermediate steps $A$ to $B$ and $A$ to $C$ $(p=0.001$ and $<0.001$, respectively). There was no difference in percentage of subjects treated with ICS in different quadrants. COPD, chronic obstructive pulmonary disease; noCOPD, without COPD; COPD, with COPD; ICS, inhaled corticosteroid; LymphoCoDe, Lymphocyte Count and Decline; BL, blood lymphocytes. 
The importance not only of BL number but also of $\mathrm{BL}$ decline in determining COPD and noCOPD outcomes is highlighted in the four-quadrant diagram we refer as LymphoCoDe (Lymphocyte Count and Decline, Fig. 4). As BL number decreases and BL decline worsens from A to $\mathrm{D}$, it can be appreciated that all clinical outcomes, from dyspnea to survival, significantly deteriorate. Importantly, progression from A to D is clearly independent of the GOLD functional severity, suggesting that the association between low-BL and worse outcomes is beyond the severity of airflow obstruction. Although count and change in BL number could be considered and plausibly used as biomarkers for COPD outcomes, it is also possible that they might play a mechanistic role in outcome deterioration.

It is important to notice that not all subjects with initial low-BL had a faster decline: about $50 \%$ of them did not decline or had an increase in BL numbers and better outcomes. A possible explanation is that the homeostatic lymphopenia-induced proliferation (LIP) [31,32], a complex physiological response that leads to expansion and restoration of T-cells during lymphopenic conditions, probably preserves lymphocyte numbers in these subjects, a mechanism that fails in subjects who have fast decline in spite of having low lymphocytes. IL-7, a cytokine produced by epithelial and endothelial cells in lymphoid organs, constitutes one of the key cues controlling LIP through signaling to naïve and memory $\mathrm{T}$-cells expressing the IL-7Receptor (IL-7R) [31-33].

LIP might be compromised when the availability of IL-7 or IL-7R expression is reduced, as it can be seen in presence of chronic immune activation [31, 34], a situation akin to COPD [9]. Furthermore, T-cells expressing programmed cell death protein-1 (PD-1) do not undergo LIP. On the other hand, PD-1 blockade could facilitate LIP and improve the numbers and effector functions of T-cells against tumors $[31,35,36]$. Possibly these facts might account for the high incidence of tumors and increased mortality seen in smokers with low-BL count and BL decay over time.

Lymphopenia has been described as an important feature in autoimmune diseases, where it might play a mechanistic role [37]. In systemic lupus erythematosus, lymphopenia can predict flares [38] and is associated with decreased survival [39], underlying the similarities between COPD, a disease with a strong adaptive immune component [9], and other autoimmune disorders. The decreased $\mathrm{CD} 4^{+}$and $\mathrm{B}$ lymphocytes, the increased $\mathrm{CD} 8^{+}$ and $\mathrm{NK}$ cells, and the reduced $\mathrm{CD} 4^{+} / \mathrm{CD}^{+}$ratio found in COPD along with the increased CD28- T-cells, have been associated with mortality in the elderly, and probably represent significant risk factors to the described outcomes in COPD [23, 40].

Being a single-center study could be a possible limitation of our study. On the other hand, having a population of smokers carefully followed longitudinally by the same group of physicians ensures an evenness in the data collection. Further larger multicenter studies would be informative about the applicability of our findings. A further limitation was the low number of patients in which lymphocyte subpopulations were characterized. A larger sample might have given a better information about the mechanisms related to the BL behavior in this population.

The mechanism by which BL changes could influence outcomes in smokers, not only in the lung but systemically, is not clear and will require a detailed study of the mechanisms inducing lymphocyte decline and the lack of compensation by LIP. Insights into these fields could improve the understanding of the mechanisms of COPD, which might guide the development of new therapies.

\section{Conclusion}

In conclusion, $\mathrm{BL}$ count and $\mathrm{BL}$ decline are related to worse outcomes in smokers with COPD and without COPD, which suggests that BL count and decline might play a mechanistic role in outcomes deterioration. Insights into mechanisms inducing the fall in BL count could improve the understanding of COPD pathogenesis and point toward new therapeutic measures. Therapies aimed at the restoration of the $\mathrm{T}$-cell repertoire might be a future plausible consideration to explore for high-risk cases.

\section{Acknowledgements}

The authors thank Dr. Giorgia Pantano from the Department of Women's and Children's Health for her help with the FACS analysis.

\section{Statement of Ethics}

The study conformed to the Declaration of Helsinki. This study was approved by human-research review board (IRB.12/2010), and all patients provided informed written consent before any procedure was done. 


\section{Conflict of Interest Statement}

The authors have no conflicts of interest to declare.

\section{Funding Sources}

The research described here was supported by grants (BIRD194033 and BIRD199551/19) from the University of Padova.

\section{Author Contributions}

U.S.; D.B.; J.M.M.; M.S.; and M.G.C.: contributed to conception and design of the study. Drafting and editing the manuscript. E.B.; B.B.; and A.C.: performed experimental work and data analysis. U.S.; M.T.; S.J.C; P.C.; and M.M-O: contributed to sample collection; undertook data collection and performed data analysis. S.B and G.T: data management and data interpretations. All the authors critically revised the manuscript for important intellectual content and approved the final version of the manuscript.

\section{References}

1 Global strategy for the diagnosis, management and prevention of chronic obstructive pulmonary disease, global initiative for chronic obstructive lung disease (GOLD). 2020. https://goldcopd.org.

2 Finkelstein R, Fraser RS, Ghezzo H, Cosio MG. Alveolar inflammation and its relation to emphysema in smokers. Am J Respir Crit Care Med. 1995;152(5 Pt 1):1666-72.

3 Saetta M, Baraldo S, Corbino L, Turato G, Braccioni F, Rea F, et al. CD8+ve cells in the lungs of smokers with chronic obstructive pulmonary disease. Am J Respir Crit Care Med. 1999;160(2):711-7.

4 Turato G, Zuin R, Miniati M, Baraldo S, Rea F, Beghé B, et al. Airway inflammation in severe chronic obstructive pulmonary disease: relationship with lung function and radiologic emphysema. Am J Respir Crit Care Med. 2002;166(1):105-10.

5 Lehmann C, Wilkening A, Leiber D, Markus A, Krug N, Pabst R, et al. Lymphocytes in the bronchoalveolar space reenter the lung tissue by means of the alveolar epithelium, migrate to regional lymph nodes, and subsequently rejoin the systemic immune system. Anat Rec. 2001;264(3):229-36.

6 Fabbri LM, Rabe KF. From COPD to chronic systemic inflammatory syndrome? Lancet. 2007;370(9589):797-9.

7 Gan WQ, Man SF, Senthilselvan A, Sin DD. Association between chronic obstructive pulmonary disease and systemic inflammation: a systematic review and a meta-analysis. Thorax. 2004;59(7):574-80.

8 Corre F, Lellouch J, Schwartz D. Smoking and leucocyte-counts. Results of an epidemiological survey. Lancet. 1971;2(7725):632-4.

9 Cosio MG, Saetta M, Agusti A. Immunologic aspects of chronic obstructive pulmonary disease. N Engl J Med. 2009;360(23):2445-54.

10 Baraldo S, Balestro E, Bazzan E, Tiné ME, Biondini D, Turato G, et al. Alpha-1 antitrypsin deficiency today: new insights in the immunological pathways. Respiration. 2016; 91(5):380-5.

11 Suzuki M, Sze MA, Campbell JD, Brothers JF, Lenburg ME, McDonough JE, et al. The cellular and molecular determinants of emphysematous destruction in COPD. Sci Rep. 2017;7(1):9562.
12 Tollerud DJ, Clark JW, Brown LM, Neuland CY, Mann DL, Pankiw-Trost LK, et al. The effects of cigarette smoking on T cell subsets: a population-based survey of healthy Caucasians. Am Rev Respir Dis. 1989;139:1446-51.

13 Tanigawa T, Araki S, Nakata A, Kitamura F, Yasumoto M, Sakurai S, et al. Increase in memory (CD4+CD29+and CD4+CD45RO+) $\mathrm{T}$ and naive (CD4+CD45RA+)T-cell subpopulations in smokers. Arch Environ Health Int J. 1998;53:378-83.

14 Zhu X, Gadgil AS, Givelber R, George MP, Stoner MW, Sciurba FC, et al. Peripheral T cell functions correlate with the severity of chronic obstructive pulmonary disease. J Immunol. 2009;182(5):3270-7.

15 De Jong JW, Van Der Belt-Gritter B, Koëter GH, Postma DS. Peripheral blood lymphocyte cell subsets in subjects with chronic obstructive pulmonary disease: association with smoking, IgE and lung function. Respir Med. 1997;91(2):67-76.

16 Glader P, von Wachenfeldt K, Löfdahl CG. Systemic CD4+ T-cell activation is correlated with FEV1 in smokers. Respir Med. 2006; 100(6):1088-93.

17 Bender BS, Nagel JE, Adler WH, Andres R. Absolute peripheral blood lymphocyte count and subsequent mortality of elderly men: the Baltimore longitudinal study of aging. J Am Geriatr Soc. 1986;34:649-54.

18 Acanfora D, Gheorghiade M, Trojano L, Furgi G, Pasini E, Picone C, et al. Relative lymphocyte count: a prognostic indicator of mortality in elderly patients with congestive heart failure. Am Heart J. 2001;142(1):167-73.

19 Furutate R, Ishii T, Motegi T, Hattori K, Kusunoki Y, Gemma A, et al. The neutrophil to lymphocyte ratio is related to disease severity and exacerbation in patients with chronic obstructive pulmonary disease. Intern Med. 2016;55(3):223-9.

20 Halper-Stromberg E, Yun JH, Parker MM, Singer RT, Gaggar A, Silverman EK, et al. Systemic markers of adaptive and innate immunity are associated with chronic obstructive pulmonary disease severity and spirometric disease progression. Am J Respir Cell Mol Biol. 2018;58(4):500-9.
21 Tinè $\mathrm{M}$, Bazzan $\mathrm{E}$, Semenzato $\mathrm{U}$, Biondini $\mathrm{D}$, Cocconcelli G, Balestro E, et al. Heart failure is highly prevalent and difficult to diagnose in severe exacerbations of COPD presenting to the emergency department. J Clin Med. 2020; 9(8):2644.

22 Vestbo J, Rennard S. Chronic obstructive pulmonary disease biomarker(s) for disease activity needed-urgently. Am J Respir Crit Care Med. 2010;182:863-4.

23 Lin Y, Kim J, Metter EJ, Nguyen H, Truong T, Lustig A, et al. Changes in blood lymphocyte numbers with age in vivo and their association with the levels of cytokines/cytokine receptors. Immun Ageing. 2016;13:24.

24 Schaberg T, Theilacker C, Nitschke OT, Lode $\mathrm{H}$. Lymphocyte subsets in peripheral blood and smoking habits. Lung. 1997;175(6):38794.

25 Celli BR, Cote CG, Marin JM, Casanova C, Montes de Oca M, Mendez RA, et al. The body-mass index, airflow obstruction, dyspnea, and exercise capacity index in chronic obstructive pulmonary disease. N Engl J Med. 2004;350(10):1005-12.

26 Warny M, Helby J, Nordestgaard BG, Birgens $\mathrm{H}$, Bojesen SE. Incidental lymphopenia and mortality: a prospective cohort study. CMAJ. 2020;192(2):E25-33.

27 Venkatesulu BP, Mallick S, Lin SH, Krishnan S. A systematic review of the influence of radiation-induced lymphopenia on survival outcomes in solid tumors. Crit Rev Oncol Hematol. 2018;123:42-51.

28 Ray-Coquard I, Cropet C, Van Glabbeke M, Sebban C, Le Cesne A, Judson I, et al. Lymphopenia as a prognostic factor for overall survival in advanced carcinomas, sarcomas, and lymphomas. Cancer Res. 2009;69(13): 5383-91.

29 Ruffell B, DeNardo DG, Affara NI, Coussens LM. Lymphocytes in cancer development: polarization towards pro-tumor immunity. Cytokine Growth Factor Rev. 2010;21(1):310.

30 McMahon LJ, Thomson SP, Nugent CA, Denny WF, Miller TP. Persistent lymphocytopenia as a diagnostic feature of bronchogenic carcinoma. Chest. 1980;78(4):583-6. 
31 Ellestad KK, Anderson CC. Two strikes and you're out? The pathogenic interplay of coinhibitor deficiency and lymphopenia-induced proliferation. J Immunol. 2017;198:2534-41.

32 Takada K, Jameson SC. Naive T cell homeostasis: from awareness of space to a sense of place. Nat Rev Immunol. 2009;9(12):823-32.

33 Barata JT, Durum SK, Seddon B. Flip the coin: IL-7 and IL-7R in health and disease. Nat Immunol. 2019;20(12):1584-93.

34 Kim HR, Hong MS, Dan JM, Kang I. Altered IL-7Ralpha expression with aging and the potential implications of IL-7 therapy on CD8+ T-cell immune responses. Blood. 2006;107(7): 2855-62.
35 Ménétrier-Caux C, Ray-Coquard I, Blay JY, Caux C. Lymphopenia in cancer patients and its effects on response to immunotherapy: an opportunity for combination with cytokines? J Immunother Cancer. 2019;7(1):85.

36 Ellestad KK, Lin J, Boon L, Anderson CC. PD-1 controls tonic signaling and lymphopenia-induced proliferation of T lymphocytes. Front Immunol. 2017;8:1289.

37 Merayo-Chalico J, Rajme-López S, BarreraVargas A, Alcocer-Varela J, Díaz-Zamudio M, Gómez-Martín D. Lymphopenia and autoimmunity: a double-edged sword. Hum Immunol. 2016;77(10):921-9.
38 Mirzayan MJ, Schmidt RE, Witte T. Prognostic parameters for flare in systemic lupus erythematosus. Rheumatology (Oxford). 2000; 39(12):1316-9.

39 Halberg P, Alsbjørn B, Balslev JT, Lorenzen I, Gerstoft J, Ullman S, et al. Systemic lupus erythematosus. Follow-up study of 148 patients. II: predictive factors of importance for course and outcome. Clin Rheumatol. 1987;6(1):226.

40 Huppert F, Pinto EM, Morgan K, Brayne C. Survival in a population sample is predicted by proportions of lymphocyte subsets. Mech Ageing Dev. 2003;124(4):449-51. 